EPJ Web of Conferences 81, 04003 (2014)

DOI: $10.1051 /$ epjconf/ 20148104003

(C) Owned by the authors, published by EDP Sciences, 2014

\title{
Heavy meson production at HERA
}

\author{
Jan Figiel ${ }^{1, a}$
}

for the $\mathrm{H} 1$ and ZEUS Collaborations

${ }^{1}$ The Henryk Niewodniczański Institute of Nuclear Physics, Polish Academy of Sciences, Kraków

\begin{abstract}
The copious production of charm mesons at HERA has allowed QCD to be probed in open charm and charm vector meson production. Elastic and proton-dissociatve photoproduction of $J / \psi$ mesons have been measured at HERA. The data are compared to other measurements and the $W$ and $t$ dependences are parametrised using phenomonological fits. The $\psi(2 S)$ state has also been measured exclusively and the ratio of its production rate to $J / \psi$ mesons presented as a function of the kinematic variables. Inelastic production of $J / \psi$ and $\psi(2 S)$ mesons gives insight into non-relativistic QCD and final results are presented here. Open charm production has been measured in order to better understand the fragmentation process of charm mesons as well as giving insight into the structure of the proton.
\end{abstract}

\section{Introduction}

Still 7 years after closing HERA ep collider new physical results based on its data are published. Here the recent, selected results on heavy meson production at HERA from the H1 and ZEUS experiments are presented and interpreted within the framework of Quantum Chromodynamics.

\section{Diffractive charmonium production}

In HERA kinematics the diffractive coherence condition is fulfilled at low Bjorken $x$ and diffraction contributes significantly to quasi-photoproduction $\left(Q^{2} \simeq 0\right)$ but also to deep inelastic scattering (DIS), at large photon virtuality $Q^{2}$. If the virtual photon fluctuates into vector meson (VM) and proton remains intact one calls this elastic VM production and when it dissociates into low-mass nucleon state $Y$ one calls it proton-dissociative one. The "elastic" VM production is quite similar to elastic hadron-hadron interaction and can be interpretted in the framework of the Regge model, via exchange of the Pomeron trajectory. In this case typical features of the "soft" processes are expected, namely weak energy dependence and steep, exponential fall-off with squared four-momentum transfer $t$, of the cross section. However if $Q^{2}$ or VM mass (as in case of charmonium) are large the process becomes "hard" and can be described within perturbative QCD via exchange of 2 gluons or gluon ladder. In this case the cross section is proportional to squared gluon density at small $x$ which translates into its strong energy dependence. Additionally, small transverse size of the $q \bar{q}$ pair results in flatter $t$-dependence of the cross section. VM production at HERA is a testbed of QCD scales and allows observation

\footnotetext{
ae-mail: jan.figiel@ifj.edu.pl
} 

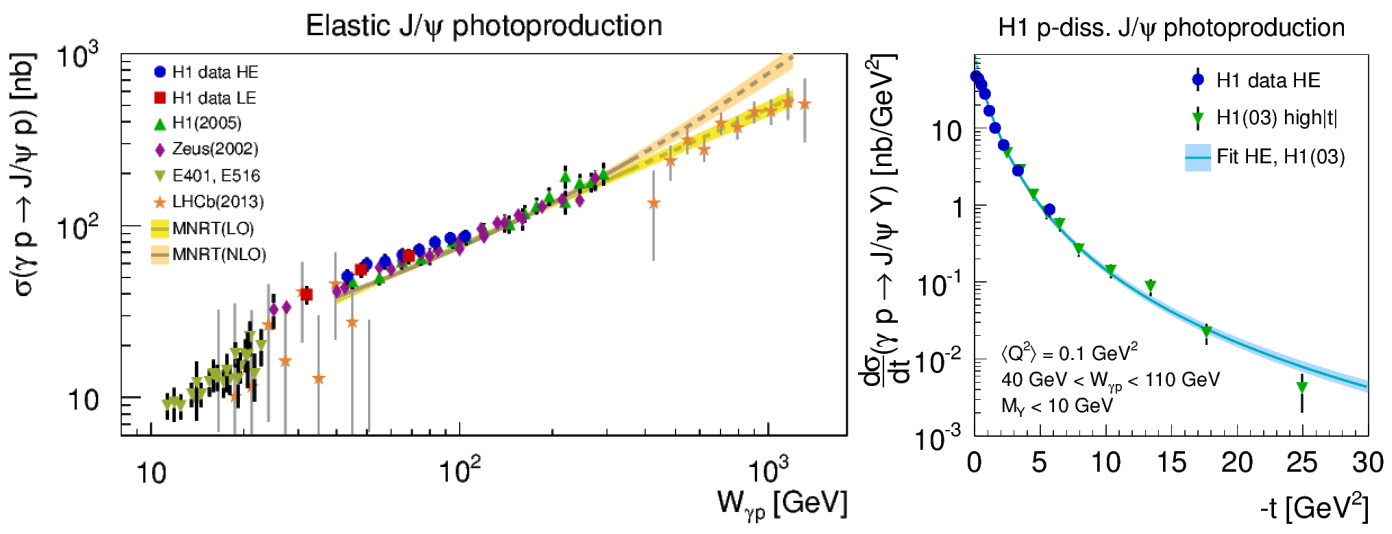

Figure 1. Left: compilation of elastic $J / \psi$ production cross section measurements as a function of energy $W$ including this measurement, previous HERA results, results from fixed target experiments and from LHCb. Also presented are QCD fits from to the previous HERA data to determine a gluon density at leading-order and next-toleading order, indicated by the curves. The fits are extrapolated from the range of the input data to higher values, as shown by the dashed curves (see [1] for references). The shaded bands indicate the fit uncertainties. Right: proton-dissociative cross section as a function of $-t$ (full circles) compared to a previous $\mathrm{H} 1$ measurement at high $|t|$. The curve represents a simultaneous power-law fit to both data sets, the shaded band shows its uncertainty.

of the transition between soft and hard regime. At larger momentum transfer $|t|>1 \mathrm{GeV}^{2}$ protondissociative process dominates. In case of charmonium production this is a two-scale process with large $J / \psi$ mass and momentum transfer $t$ and in the leading logarithmic (LL) QCD approximation it can be described by the exchange of a gluon ladder. So at large $|t|$ values, the cross section is expected to decrease according to power law (in contrast to "elastic" VM production, where exponential $t$ dependence is observed).

Recently, new, based on full HERA data, analysis of elastic and proton-dissociative photoproduction of $J / \psi$ mesons was published by the H1 Collaboration [1]. Here we show as examples the energy $W$ dependence of the elastic $J / \psi$ photoproduction and $|t|$ dependence of proton-dissociative production (figure 1). As can be seen from the figure, data behave as expected within QCD framework: elastic cross section rises fast with increasing energy, the perturabtive QCD calculations reproduce this rise and $|t|$ distribution in proton-dissociative photoproduction follows power law.

Elastic production of heavier charmonium was also studied; the ZEUS Collaboration presented results on $\psi(2 S)$ production ([2]). As $\psi(2 S)$ wave function has node at $r=0.4 \mathrm{fm}$ one expects in particular that the ratio $R=\frac{\sigma(\psi(2 S))}{\sigma(J / \psi)}$ increases with $Q^{2}$ and is independent of $W$ and $|t|$. This turned out to be the case: for example, in figure 2 , the $Q^{2}$ dependence of this ratio is shown.

\section{Inelastic $J / \psi$ and $\psi(2 S)$ production}

At HERA also inelastic charmonia production is observed. In LO QCD approximation this process may be described via Color Singlet (CS) mechanism, when $J / \psi$ quantum numbers are the same as those of charmed quark pair or via Color Octet (CO) one, when they are different. Recently ZEUS Collaboration published results on inelastic $J / \psi$ and $\psi(2 S)$ production ([3]) and compared them with 


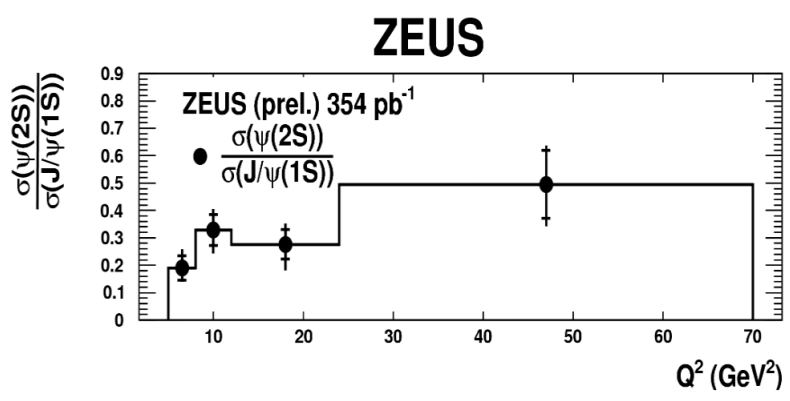

Figure 2. The ratio of cross sections for elastic electroproduction of $\psi(2 S)$ and $J / \psi R=\frac{\sigma(\psi(2 S))}{\sigma(J / \psi)}$ as a function of $Q^{2}$.

the above mentioned models: it turned out that CS model provides better description of the experimental reslts than $\mathrm{CO}$ one.

\section{Open charm production}

In ep collisions open charm is produced predominantly by boson-gluon fusion and contributes up to $30 \%$ of DIS cross section. Its description is a challenge for $\mathrm{pQCD}$ as it is sensitive to various QCD scales, $c$-quark mass, gluon density in the proton (PDFs) and used mass schemes in NLO calculations. It is however promising field as well, as measurement of charmed hadrons allows to test particular pQCD calculations, to test universality of heavy quark fragmentation and to extract charm contribution to proton structure function. For example ZEUS experiment measured $D^{ \pm}$production in DIS and confronted successfully with NLO QCD calculations based on FFNS mass scheme (HVQDIS) ([4]).

Universality of charmed quark fragmentation in various interactions was also experimentally confirmed ([5]).

Both experiments, H1 and ZEUS, measured production of various charmed mesons in DIS using different charm tagging. They combined their data using advanced method yielding results of about 2 times lower uncertainty than for each most precise input data set ([6]). The measurements were extrapolated to the full phase space using an NLO QCD calculation to obtain the reduced charm quark-pair cross sections in the region of photon virtualities $2.5 \leq Q^{2} \leq 2000 \mathrm{GeV}^{2}$. The combined charm cross sections were compared to QCD predictions using different implementations of the flavour-number-scheme.The best description of the data in the whole kinematic range was provided by the NNLO fixed-flavour-number-scheme prediction. Using the combined charm cross sections together with the combined HERA inclusive DIS data, an NLO QCD analysis was performed based on different implementations of the variable-flavour-number-scheme, with optimal choice of charm mass parameter $M_{c}$. Example of such analysis is shown in figure 3 (see caption for details). As can be seen from the figure these NLO predictions describe the charm data well which means that pQCD yields consistent picture of proton structure.

\section{Summary}

HERA was heavy meson factory. Diffractive and inelastic charmonium production as well as open charm production are described reasonably within $\mathrm{pQCD}$ framework; charm contribution to proton structure function is understood too. 


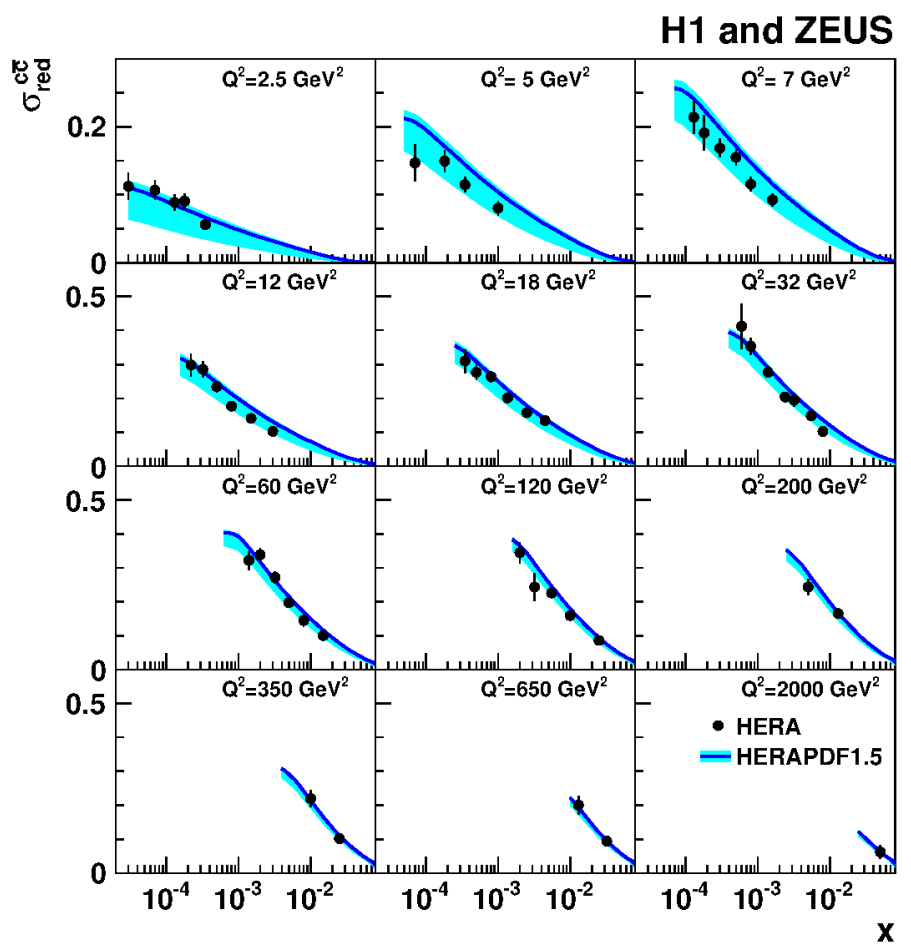

Figure 3. Combined charm reduced cross sections $\sigma_{r e d}^{c c}$ (filled circles) as a function of $x$ for fixed values of $Q^{2}$. The error bars represent the total uncertainty including uncorrelated, correlated and procedural uncertainties added in quadrature. The data are compared to the NLO predictions based on HERAPDF1.5 extracted in the RT standard scheme. The line represents the prediction using $M_{c}=1.4 \mathrm{GeV}$. The uncertainty band shows the full PDF uncertainty which is dominated by the variation of $\mathrm{M}([6])$.

\section{Acknowledgements}

I would like to thank colleagues from the H1 and ZEUS Collaborations for fruitful discussions.

\section{References}

[1] C. Alexa et al. (H1 Collaboration), Eur. Phys. J. C73, 2466 - 2500, (2013)

[2] N. Kovalchuk for the ZEUS Collaboration, XXII International Workshop on Deep Inelastic Scattering and Related Subjects, Warsaw, 28.04 - 2.05.2014

[3] H. Abramowicz et al. (ZEUS Collaboration), JHEP 02, 071 - 100 (2013)

[4] H. Abramowicz et al. (ZEUS Collaboration), JHEP 05, 023 - 052 (2013)

[5] H. Abramowicz et al. (ZEUS Collaboration), JHEP 09, 058 - 085 (2013)

[6] F. D. Aaron et al. (H1 and ZEUS Collaborations), Eur. Phys. J. C73, 2311 - 2336, (2013) 